\title{
Investigation into cardiac sympathetic innervation during the commencement of haemodialysis in patients with chronic kidney disease
}

Walter Noordzij ${ }^{1 *}$ (D, Akin Özyilmaz ${ }^{2,3}$, Andor W. J. M. Glaudemans ${ }^{1}$, René A. Tio ${ }^{4}$, Esther R. Goet ${ }^{2,3}$, Casper F. M. Franssen ${ }^{2}$ and Riemer H. J. A. Slart ${ }^{1}$

\begin{abstract}
Background: Patients with chronic kidney disease (CKD) who undergo chronic haemodialysis (HD) show altered sympathetic tone, which is related to a higher cardiovascular mortality. The purpose of this study was to investigate the effect of transition from pre-HD to HD on cardiac sympathetic innervation.

Methods: Eighteen patients aged $58 \pm 18$ years (mean \pm standard deviation [SD]), 13 males and five females, with stage 5 CKD and nine healthy control subjects aged $52 \pm 17$ (mean \pm SD), three males and six females, were included in this prospective study between May 2010 and December 2013. All patients underwent ${ }^{123}$-labelled meta-iodobenzylguanidine $\left({ }^{123}\right.$ I-MIBG) scintigraphy for cardiac sympathetic innervation and electrocardiographically gated adenosine stress and rest ${ }^{99 \mathrm{~m}} \mathrm{Tc}$-labelled tetrofosmin single-photon emission computed tomography for myocardial perfusion imaging prior to (pre-HD) and 6 months after the start of HD. Results of ${ }^{123}$ I-MIBG scans in patients were compared to controls. Impaired cardiac sympathetic innervation was defined as late heart-to-mediastinum ratio $(\mathrm{HMR})<2.0$.

Results: Mean late HMR was lower in patients during HD (2.3) than in controls (2.9) ( $p=0.035)$; however, in patients it did not differ between pre-HD and after the start of HD. During HD, two patients showed new sympathetic innervation abnormalities, and in three patients innervation abnormalities seemed to coincide with myocardial perfusion abnormalities.

Conclusions: CKD patients show cardiac sympathetic innervation abnormalities, which do not seem to progress during the maintenance HD. The relationship between sympathetic innervation abnormalities and myocardial perfusion abnormalities in HD patients needs further exploration.
\end{abstract}

Keywords: Chronic kidney disease, Haemodialysis, Myocardial ischaemia, Myocardial scintigraphy, Sympathetic innervation

\footnotetext{
* Correspondence: w.noordzij@umcg.nl

${ }^{1}$ Department of Nuclear Medicine and Molecular Imaging, University of

Groningen, University Medical Center Groningen, Groningen, The

Netherlands

Full list of author information is available at the end of the article
} 


\section{Key points}

- End-stage renal disease patients on maintenance haemodialysis have lower mean late heart-tomediastinum ratio on ${ }^{123}$ I-labelled metaiodobenzylguanidine scintigraphy than healthy control subjects

- Mean late heart-to-mediastinum ratio does not seem to worsen during the commencement of maintenance haemodialysis

- Patients with signs of cardiac sympathetic innervation abnormalities before the start of haemodialysis developed alterations in myocardial perfusion during haemodialysis

\section{Background}

Chronic kidney disease (CKD) patients who undergo haemodialysis (HD) show increased sympathetic tone and increased serum norepinephrine levels [1-3]. In addition, myocardial blood flow decreases significantly during $\mathrm{HD}$, indicating that acute HD-associated factors play a role in myocardial perfusion abnormalities $[4,5]$. In normal population, cardiac ischaemia and autonomic dysfunction are reliable predictors for future cardiac events and are also associated with increased long-term mortality [6-8]. The presence of these findings in CKD patients is related to a higher cardiovascular mortality, exceeding that of age- and gender-matched healthy control subjects [9].

An unknown number of individuals with CKD are completely asymptomatic for coronary artery disease (CAD). Especially CKD patients with concomitant diabetes show silent myocardial ischaemia and diabetic autonomic neuropathy. However, both the extent of cardiac ischaemia and sympathetic innervation abnormalities as well as the timepoint of their development during or even before starting HD are unclear. It was previously shown that cardiomyocytes are more vulnerable to sympathetic innervation abnormalities than to ischaemia, which may indicate that cardiac sympathetic innervation abnormalities precede myocardial ischaemia, [10]. Autonomic dysfunction is also commonly present in end-stage renal disease [11], but whether this results in lack of symptoms in cardiac ischaemia is unknown.

Thus, CKD patients undergoing HD show sympathetic innervation abnormalities. At present, it is unclear whether these abnormalities are present before the start of $\mathrm{HD}$ or occur during the commencement of maintenance HD. So far, the presence and severity of cardiac sympathetic innervation abnormalities and CAD has never been studied in asymptomatic end-stage (stage 5) CKD patients who are not yet on HD. Therefore, the aim of this study is to determine baseline cardiac sympathetic innervation in asymptomatic stage 5 CKD patients, and to investigate the early effect of the initiation of maintenance HD on cardiac sympathetic innervation.

\section{Methods \\ Patients}

This study was approved by the institutional ethics review board ('medisch ethische toetsingscommissie' of the University Medical Center Groningen, chaired by professor WA Kamps, protocol number 2008/133, date of approval June 1, 2008). All included patients gave their written informed consent for participation in this study. Patient information was anonymised and de-identified before data analysis.

Between May 2010 and December 2013 a total of 18 consecutive patients (13 males and five females) with stage 5 CKD were prospectively included. Diagnosis of CKD was based on serum levels of creatinine, and subsequent estimated glomerular filtration rate (eGFR), the latter determined by the Modification of Diet in Renal Disease method [12]. Included were patients aged $>18$ years who had no history of CAD and were asymptomatic for present CAD, had no history of Parkinson's disease or dementia with Lewy bodies, were not currently using tricyclic antidepressant agents which could interfere with ${ }^{123}$ I-labelled meta-iodobenzylguanidine $\left({ }^{123} \mathrm{I}-\mathrm{MIBG}\right)$ uptake, and were expected to start HD within the next 6 months. All patients underwent laboratory tests at baseline. Electrocardiograms in pre-HD and during chronic HD were retrieved from the digital patient chart.

${ }^{123}$ I-MIBG scintigraphy for cardiac sympathetic innervation and ${ }^{99 \mathrm{~m}}$ Tc-tetrofosmin scintigraphy for myocardial perfusion imaging were performed before the start of HD and 6 months after the start of HD. Patients who showed CAD in pre-HD were not excluded from the study to investigate the progression of CAD during maintenance HD. All scans were performed on interdialytic days: a non-weekend day before or after an HD day. Consensus reading on myocardial perfusion scans was performed during general patient care board meetings. All scans were reviewed by an experienced nuclear medicine physician (RHJAS, with $>20$ years experience), and cardiologist (RAT, with $>20$ years experience).

For ${ }^{123}$ I-MIBG imaging, nine age-matched normal volunteers were scanned, each on one occasion, to collect a healthy control database. All subjects were in good health, i.e. they did not suffer from known (current or previously treated) hypertension, heart disease, diabetes mellitus, or renal impairment and did not use medication.

\section{Haemodialysis}

Patients were dialyzed three times per week. All patients were on bicarbonate dialysis with a low-flux polysulfone hollow-fiber dialyser F8 (Fresenius Medical Care, Bad Hamburg, Germany). Blood flow and dialysate flow rates 
were $250-350 \mathrm{ml} / \mathrm{min}$ and $500 \mathrm{ml} / \mathrm{min}$, respectively. Dialysate temperature was 36.0 or $36.5{ }^{\circ} \mathrm{C}$. Dialysate composition was sodium $139 \mathrm{mmol} / \mathrm{l}$, potassium 1.0 or $2.0 \mathrm{mmol} / \mathrm{l}$, calcium $1.5 \mathrm{mmol} / \mathrm{l}$, magnesium $0.5 \mathrm{mmol} / \mathrm{l}$, chloride $108 \mathrm{mmol} / \mathrm{l}$, bicarbonate $34 \mathrm{mmol} / \mathrm{l}$, acetate $3.0 \mathrm{mmol} / \mathrm{l}$ and glucose $1.0 \mathrm{~g} / \mathrm{dl}$.

\section{${ }^{123}$ I-MIBG scintigraphy}

Scintigraphy was performed after blockade of thyroid uptake of free ${ }^{123}$ I by iodine-potassium iodide (Lugol's solution). After a 15-min resting period, subjects were injected with $185 \mathrm{MBq}(5 \mathrm{mCi}){ }^{123} \mathrm{I}$-MIBG (General Electric Healthcare Medical Diagnostics, Eindhoven, The Netherlands) by an intravenous catheter. At $15 \mathrm{~min}$ (early image) and $4 \mathrm{~h}$ (late image) after tracer administration, a 10 -min static anterior view of the chest was acquired using a Symbia S gamma camera (Siemens Medical System, Knoxville, Tennessee, USA) with a medium-energy low-penetration parallel-hole collimator [13]. A $15 \%$ energy window centred on $159 \mathrm{keV}$, a $256 \times 256$ matrix size and a 1.45 zoom factor were used. According to guidelines for ${ }^{123}$ I-MIBG scintigraphy, the use of $\beta$-adrenoceptor blocking agents ( $\beta$-blockers) was not discontinued [13].

For planar images, left ventricle (LV) activity was measured over the raw static image using a region of interest along the contour of the LV. A second region of interest was placed over the upper mediastinum [13]. The heartto-mediastinum ratio (HMR) was measured three times, and the average of measurements was taken into account. Late HMR $<2.0$ was considered as a sign of cardiac sympathetic innervation abnormalities [13]. Data were also compared with our local normal database.

\section{${ }^{99} \mathrm{~m}$ Tc-tetrofosmin SPECT}

Adenosine stress ${ }^{99} \mathrm{~m}$ Tc-tetrofosmin $(250 \mathrm{MBq}, 6.8 \mathrm{mCi})$ and rest ${ }^{99 \mathrm{~m}} \mathrm{Tc}$-tetrofosmin $(750 \mathrm{MBq}, 20 \mathrm{mCi})$ electrocardiographically gated single-photon emission computed tomography (SPECT) were performed in consecutive order in a 1-day protocol to analyse myocardial ischaemia. Patients were asked to withdraw caffeine-containing beverages and/or food $24 \mathrm{~h}$ before the examination. ${ }^{99 \mathrm{~m}} \mathrm{Tc}$-tetrofosmin SPECT studies were obtained $1 \mathrm{~h}$ after tracer administration using a dual-headed gamma camera, equipped with low-energy high-resolution collimators (Symbia T16 gamma camera, Siemens Medical System, Knoxville, Tennessee, USA), electrocardiographic gating, and low-dose computed tomography for attenuation correction. All data from the ${ }^{99 \mathrm{~m}} \mathrm{Tc}$-tetrofosmin SPECT studies were reformatted to obtain short-axis, horizontal and vertical long-axis sections. Data were analyzed and displayed in a 17-segment polar map using the Quantitative Perfusion SPECT application, a commercially available gated cardiac software package developed by the
Cedars-Sinai Medical Center (Los Angeles, CA, USA) [14]. Average counts per segment were obtained from the 17 segments and the measured counts were normalized to the segment with the highest average counts.

\section{Statistical analysis}

Baseline descriptive statistics are presented as mean \pm standard deviation or median (range) for continuous variables and numbers with percentages for categorical variables as required. We evaluated differences between the two study groups using the $x^{2}$ test and Fisher exact test for categorical data and the Student $t$-test and Mann-Whitney $U$ test for continuous data, according to whether data were normally distributed. Furthermore, a two-way ANOVA with Bonferroni correction for multiple comparison was performed to analyse the differences in study groups over time. In all analyses, $p<0.050$ was considered statistically significant. Statistical analysis was performed using the SPSS package version 22 (IBM Corp., Armonk, NY, USA).

\section{Results}

\section{Patient characteristics}

Baseline characteristics of the patients are summarized in Table 1 . The mean age was $58 \pm 18$ years. For the nine healthy controls (three males and six females), the age (52 \pm 17 years) was not significantly different $(p=0.423)$. As expected in patients with stage 5 CKD, eGFR indicated severely reduced kidney function $(<15 \mathrm{ml} / \mathrm{min})$ in all patients. A majority of the patients had hypertension (78\%) and used anti-hypertensive medication, mainly $\beta$-blockers and diuretics. Diabetes mellitus was present in $33 \%$ of the patients.

At baseline, none of the patients had electrocardiographic signs of ischaemia or infarction. One patient had a first degree atrio-venticular block whereas another patient had a left bundle branch block (QRS duration $138 \mathrm{~ms}$ ).

\section{${ }^{123}$ I-MIBG scintigraphy}

Table 2 shows the results of ${ }^{123}$ I-MIBG scans. All 18 patients underwent ${ }^{123}$ I-MIBG scintigraphy in pre-HD and during chronic HD. ${ }^{123}$ I-MIBG scans during chronic HD were performed within $6 \pm 2$ months (range 3-13 months) after the start of HD. Late HMR in patients in pre-HD was not different from healthy controls. There was no significant difference (especially no significant decrease) in late HMR between pre-HD and chronic HD. However, during chronic HD patients showed significant lower late HMR than healthy controls: mean late HMR 2.3 vs 2.9 $(p=0.036$; Fig. 1$)$. In pre-HD, three patients had signs of impaired cardiac sympathetic innervation, whereas five patients showed cardiac sympathetic innervation abnormalities during chronic HD. None of these five patients used angiotensin converting enzyme inhibitors. 
Table 1 Patient characteristics at baseline

\begin{tabular}{|c|c|}
\hline & $\begin{array}{l}\text { Frequency, median (range) } \\
\text { or mean } \pm \text { standard deviation }\end{array}$ \\
\hline \multicolumn{2}{|l|}{ Gender (number) } \\
\hline Male & $13(72 \%)$ \\
\hline Female & $5(28 \%)$ \\
\hline Age (years) & $58 \pm 18$ \\
\hline \multicolumn{2}{|l|}{ Medical history (number) } \\
\hline Hypertension & $14(78 \%)$ \\
\hline Hypercholesterolemia & $6(33 \%)$ \\
\hline Diabetes mellitus & $6(33 \%)$ \\
\hline Smoking & $2(11 \%)$ \\
\hline Coronary artery disease & $0(0 \%)$ \\
\hline \multicolumn{2}{|l|}{ Medication at baseline (number) } \\
\hline$\beta$-blockers & $11(61 \%)$ \\
\hline ACE ihibitors & $6(33 \%)$ \\
\hline Diuretics & $8(44 \%)$ \\
\hline \multicolumn{2}{|l|}{ Laboratory at inclusion } \\
\hline Leucocytes $\left(\times 10^{9} / \mathrm{l}\right)$ & $7.1(3.3-9.8)$ \\
\hline Haemoglobin $(\mathrm{mmol} / \mathrm{l})$ & $6.8(6.0-9.7)$ \\
\hline Trombocytes $\left(\times 10^{9} / l\right)$ & $208(117-347)$ \\
\hline C-reactive protein (mg/l) & $0(0-59)$ \\
\hline Sodium (mmol/l) & $140(135-145)$ \\
\hline Potassium (mmol/l) & $4.9(4.0-5.9)$ \\
\hline Chloride (mmol/l) & $107(98.0-114)$ \\
\hline Urea $(m m o l / l)$ & $26(15-38)$ \\
\hline Creatinine $(\mu \mathrm{mol} / \mathrm{l})$ & $584\left(339-1.31 \times 10^{3}\right)$ \\
\hline eGFR $\left(\mathrm{ml} / \mathrm{min} \times 1.73 \mathrm{~m}^{2}\right)$ & $8.0(4.0-12)$ \\
\hline Uric acid (mmol/l) & $0.45(0.23-0.72)$ \\
\hline Calcium (mmol/l) & $2.25(2.07-2.53)$ \\
\hline Phosphate (mmol/l) & $1.60(1.07-2.12)$ \\
\hline Total protein (g/l) & $67(60-77)$ \\
\hline Albumin $(g / l)$ & $41(29-46)$ \\
\hline $\begin{array}{l}\text { High-density lipoprotein cholesterol } \\
(\mathrm{mmol} / \mathrm{l})\end{array}$ & $1.0(0.70-2.7)$ \\
\hline $\begin{array}{l}\text { Low-density lipoprotein cholesterol } \\
(\mathrm{mmol} / \mathrm{l})\end{array}$ & $2.2(0.80-4.8)$ \\
\hline Glucose (mmol/l) & $6.7(4.1-14)$ \\
\hline Urinary protein excretion (g/24 h) & $2.7(0.40-6.8)$ \\
\hline
\end{tabular}

ACE angiotensin converting enzyme, eGFR estimated glomerular filtration rate

Four of these patients did use $\beta$-blockers. Only one patient with diabetes mellitus developed cardiac sympathetic innervation abnormalities during HD.

\section{${ }^{99 m}$ Tc-tetrofosmin SPECT}

The results of myocardial perfusion SPECT are summarized in Table 3. Pre-HD myocardial perfusion SPECT was performed in 17 out of 18 (94\%) patients. One
Table $2^{123}$ I-MIBG findings

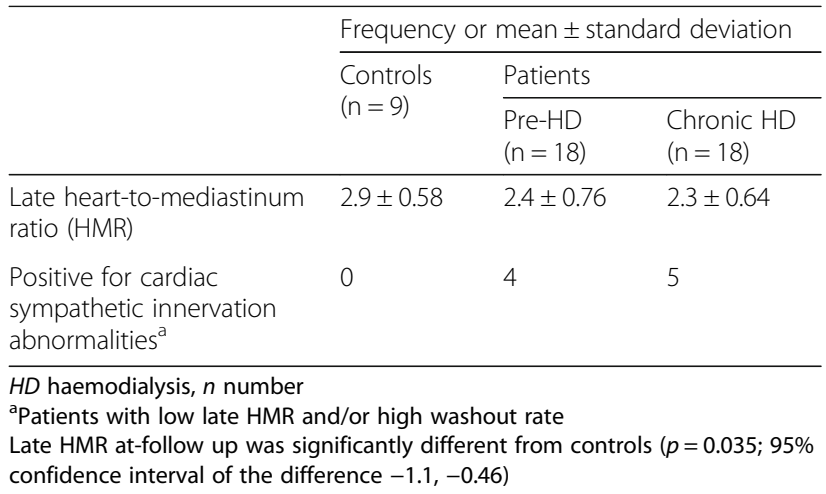

patient was not able to undergo the investigation before the start of HD. Myocardial ischaemia was present in six (35\%) patients, and five of these six patients also showed wall motion abnormalities, especially hypokinesia of the affected LV wall. None of the patients had signs of infarction in pre-HD. Mean LV ejection fraction $(56 \pm 17 \%)$ was within a normal range.

Chronic HD myocardial perfusion SPECT was performed within $6 \pm 2$ months (range 3-13 months) after the start of HD. During chronic HD, four patients showed myocardial ischaemia. Two of these four patients had developed new myocardial ischaemia during HD while in the other two patients myocardial ischaemia was already present in pre-HD. One of these latter two patients was an elderly lady with a prolonged history of diabetes mellitus. Two patients with myocardial ischaemia in pre-HD had normal myocardial perfusion SPECT results during chronic HD. Both patients with myocardial infarction during chronic HD showed progression from myocardial ischaemia in pre-HD.

During chronic HD, neither mean LV ejection fraction nor mean end-diastolic volumes were statistically different from pre-HD.

\section{Relationship between myocardial perfusion and sympathetic innervation}

Four patients already had signs of cardiac sympathetic innervation abnormalities in pre-HD. One patient with a positive ${ }^{123}$ I-MIBG scan during chronic HD developed new myocardial ischaemia, one patient developed an infarction out of ischaemia in pre-HD (Figs. 2 and 3) and one patient showed an increase in ischaemic area. Two patients with positive ${ }^{123}$ I-MIBG scans during chronic HD had normal myocardial perfusion scans in pre-HD and during chronic HD. These two patients, who showed normalization of myocardial ischaemia in pre-HD, had no signs of cardiac sympathetic innervation abnormalities in pre-HD or during chronic HD. 


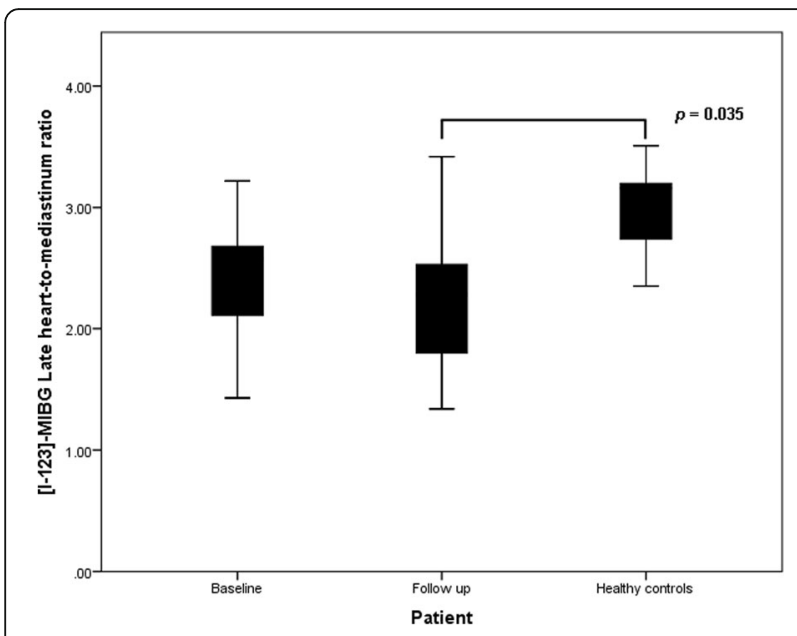

Fig. 1 Late ${ }^{123}$ I-MIBG heart-to-mediastinum ratio (HMR) in pre-HD and during chronic HD, and of healthy control subjects. Late HMR was significantly lower in patients during chronic HD than in healthy controls

\section{Discussion}

During the commencement of HD, mean late HMR was lower than in healthy controls but not compared to patients in pre-HD. The presence of cardiac sympathetic innervation abnormalities detected by ${ }^{123}$ I-MIBG scintigraphy in HD patients is not a novel concept. However, a statistical difference in HMR between patients and healthy controls has never been established before $[15,16]$, nor was it in this study. In addition, a difference in late HMR between baseline and during the commencement of maintenance HD could not be established. A previous study showed that HMR is lower in patients undergoing maintenance $\mathrm{HD}$ for a prolonged period of time, up to 120 months [17]. However, in that study ${ }^{123}$ I-MIBG scintigraphy was performed at only one single time point: the effect on HMR of transition from pre-HD to HD was not investigated. The present study provides additional information that cardiac sympathetic innervation abnormalities do not seem to worsen during the commencement of maintenance HD.

The results of this study should be interpreted with caution due to limited power. A power analysis was

Table 3 Gated myocardial perfusion SPECT results

\begin{tabular}{lll}
\hline & \multicolumn{2}{l}{ Frequency or mean \pm standard deviation } \\
\cline { 2 - 3 } & $\begin{array}{l}\text { Baseline } \\
(\mathrm{n}=17)\end{array}$ & $\begin{array}{l}\text { Follow up } \\
(\mathrm{n}=17)\end{array}$ \\
\hline $\begin{array}{l}\text { End-diastolic } \\
\text { volume }(\mathrm{ml})\end{array}$ & $137 \pm 41.2$ & $122 \pm 53.2$ \\
$\begin{array}{l}\text { Left ventricular } \\
\text { ejection fraction (\%) }\end{array}$ & $56 \pm 7.0$ & $58 \pm 7.0$ \\
ischaemia & 6 & 4 \\
Infarction & 0 & 2 \\
\hline
\end{tabular}

$n$ number performed and resulted in a required number of 44 included patients. Initially, patients with CAD detected on pre-HD myocardial perfusion SPECT were excluded from this study. Due to a very slow inclusion of patients with no CAD at all, we decided to also include patients with CAD in pre-HD. This did not result in an increase in the inclusion rate. Therefore, the study was terminated before the necessary number of patients was included. Eventually, only 18 patients completed the study protocol. If the required number of patients had been included, the results might have been more definitive. However, the present study comprises the largest group of patients in which cardiac sympathetic innervation has been evaluated during the initiation of maintenance HD.

In addition, the development of myocardial perfusion abnormalities during the commencement of maintenance HD should be interpreted with caution. Patients who showed myocardial ischaemia in pre-HD were treated with maximal anti-ischaemic medication. The affected areas were too small to refer for percutaneous intervention. We cannot be sure that treatment influenced the outcome of the scans during chronic HD. Since two out of the three patients with low late HMR in pre-HD showed alteration in myocardial perfusion from pre-HD to chronic HD, this may suggest that cardiac sympathetic denervation precedes the development of myocardial perfusion defects. This suggestion supports the fact that cardiomyocytes are more susceptible to sympathetic innervation abnormalities than to decreased blood flow [18]. Furthermore, two patients in this study also developed sympathetic denervation without myocardial perfusion abnormalities, which may suggest (not yet detectable) microvascular coronary dysfunction. However, six of the included 18 patients already showed myocardial ischaemia in pre-HD despite being asymptomatic for CAD. Then again, with the reported small areas of myocardial ischaemia and infarction in this cohort consisting of asymptomatic patients, the detection rate of myocardial perfusion SPECT was rather limited. Furthermore, cardiac magnetic resonance imaging seems to outperform SPECT for the detection of CAD [19]. The reliability of the scans of the affected ischaemic or infarcted areas could also have been improved by performing inter-observer agreements. Although experienced physicians reviewed the myocardial perfusion SPECT scans in general patient care board meetings, this consensus reading is not a substitute for inter-observer agreement analysis.

Other important issues that influence outcome are the time between baseline scans and the start of HD as well as the time between the start of HD and follow-up scans. It appeared to be very difficult to determine the time point for imaging, particularly in patients with very fragile kidney function. Some of the 


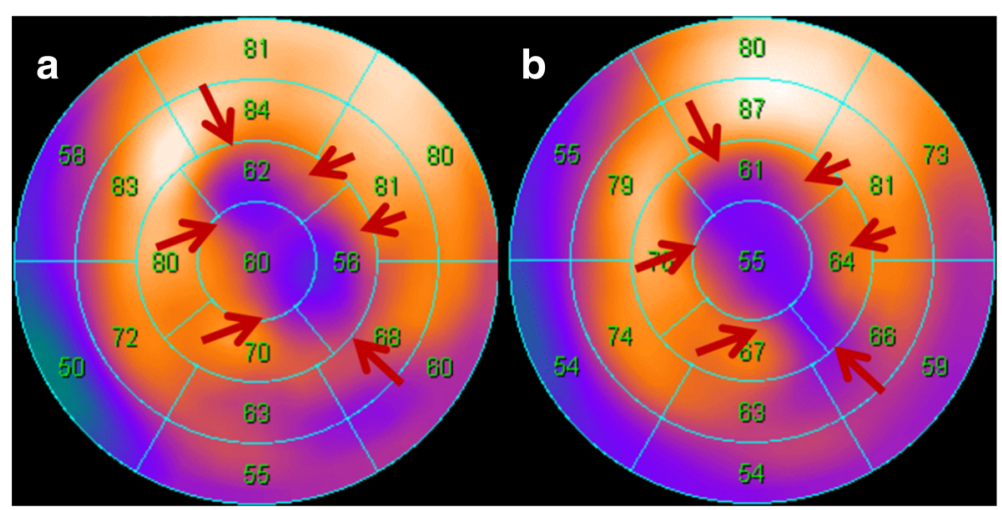

Fig. 2 Polar map reconstruction of the myocardial perfusion SPECT of a patient during chronic HD: a stress image; $\mathbf{b}$ rest image. The persisting perfusion defect in the antero-apical segments of the left ventricle wall (red arrows) indicates myocardial infarction

patients had stable CKD for a prolonged period of time but underwent sudden and rapid deterioration. There were also patients who required the start of HD to be postponed because the deterioration in kidney function was not as rapid as expected. The largest time frame between baseline to follow-up scans was 13 months. Unfortunately, the researchers were not always aware of this change in schedule. Therefore, uncertainty about the start of HD resulted in the differences in the time-interval of imaging.

According to the 2012 United States Renal Data System annual report, arrhythmia and sudden cardiac arrest accounted for $63 \%$ of the cardiovascular and $27 \%$ of all-cause mortality in dialysis patients [20]. In a recent study involving 75 HD patients with an implantable cardioverter defibrillator (ICD), nearly $80 \%$ of all sudden cardiac arrests were caused by ventricular tachycardia and ventricular fibrillation [21]. The authors reported a higher survival rate after sudden cardiac arrest than previously assumed. ${ }^{123}$ I-MIBG scans are known to have predictive implications for successful ICD therapy in patients with heart failure [22]. Therefore, ${ }^{123}$ I-MIBG scintigraphy has the potential to play a guiding role in clinical ICD decision-making in HD patients. Until that time, prospective studies have to demonstrate the relationship between low late HMR and the development of ischemic heart failure-related arrhythmia during HD.

To summarise, stage 5 CKD patients on maintenance HD showed a lower mean late HMR than healthy controls. Mean late HMR does not seem to worsen after the commencement of HD or during maintenance HD. Although present in a minority of the patients, signs of cardiac sympathetic innervation abnormalities in preHD may lead to alterations in myocardial perfusion during chronic HD. Further prospective studies should provide more insight into the long-term effect of cardiac sympathetic innervation abnormalities on the development of ischaemic heart failure-related arrhythmia during HD.
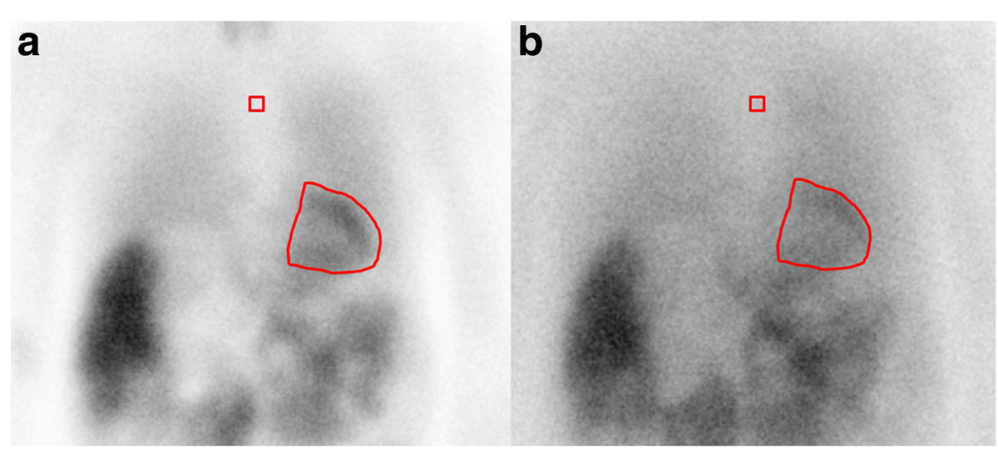

Fig. 3 Planar images from ${ }^{123}$-MIBG scintigraphy of a patient during chronic HD. a Early image 15 min after tracer administration; b late image $4 \mathrm{~h}$ after tracer administration. Late heart-to-mediastinum ratio (HMR) is the ratio between the counts within a reference point in the upper mediastinum (red square box) and the left ventricle (red contoured region). The HMR of panel a was 3.03 and of panel $\mathbf{b} 1.80$, resulting in a $41 \%$ washout 


\section{Abbreviations}

ACE: Angiotensin converting enzyme; CAD: Coronary artery disease; CKD: Chronic kidney disease; eGFR: Estimated glomerular filtration rate; HD: Haemodialysis; HMR: Heart-to-mediastinum ratio; ICD: implantable cardioverter defibrillator; LV: Left ventricle; MIBG: meta-iodobenzylguanidine; SD: Standard deviation; SPECT: Single-photon emission computed tomography

\section{Acknowledgements}

We would like to thank Herma Uiterwijk for her contribution in daily patient care. We also would like to thank GE Healthcare for providing [123I]-MIBG radiopharmaceuticals during follow-up and for healthy control subjects.

\section{Authors' contributions}

WN scans analysis, statistical analysis, writing the manuscript. AO screening patients, inclusion of patients, editing manuscript. AWJMG editing the manuscript. RAT design of the study, scan analysis, editing the manuscript. ERG screening and inclusion of patients. CFMF design of the study, editing of the manuscript. RHJAS design of the study, scan analysis, editing the manuscript. All authors read and approved the final manuscript.

\section{Competing interests}

The authors declare that they have no competing interests.

\section{Publisher's Note}

Springer Nature remains neutral with regard to jurisdictional claims in published maps and institutional affiliations.

\begin{abstract}
Author details
${ }^{1}$ Department of Nuclear Medicine and Molecular Imaging, University of Groningen, University Medical Center Groningen, Groningen, The Netherlands. ${ }^{2}$ Division of Nephrology, Department of Internal Medicine, University of Groningen, University Medical Center Groningen, PO Box 30.001, 9700 RB Groningen, The Netherlands. Dialysis Center Groningen, University of Groningen, University Medical Center Groningen, PO Box 30.001, 9700 RB Groningen, The Netherlands. 'Department of Cardiology, University of Groningen, University Medical Center Groningen, PO Box 30.001, 9700 RB Groningen, The Netherlands.
\end{abstract}

Received: 8 August 2017 Accepted: 20 October 2017

Published online: 06 December 2017

\section{References}

1. Ewing DJ, Winney R (1975) Autonomic function in patients with chronic renal failure on intermittent haemodialysis. Nephron 15:424-429

2. Ritz E, Deppisch R, Stier E, Hänsch G (1994) Atherogenesis and cardiac death: are they related to dialysis procedure and biocompatibility? Nephrol Dial Transplant 9:165-172

3. Tonelli M, Wiebe N, Culleton B et al (2006) Chronic kidney disease and mortality risk: a systematic review. J Am Soc Nephrol 17:2034-2047

4. McIntyre CW, Burton JO, Selby NM et al (2008) Hemodialysis-induced cardiac dysfunction is associated with an acute reduction in global and segmental myocardial blood flow. Clin J Am Soc Nephrol 3:19-26

5. Dasselaar JJ, Slart RH, Knip M et al (2009) Haemodialysis is associated with a pronounced fall in myocardial perfusion. Nephrol Dial Transplant 24:604-610

6. Biagini $\mathrm{E}$, Schinkel AF, Bax JJ et al (2005) Long term outcome in patients with silent versus symptomatic ischaemia during dobutamine stress echocardiography. Heart 91:737-742

7. Laukkanen JA, Kurl S, Lakka TA et al (2001) Exercise-induced silent myocardial ischemia and coronary morbidity and mortality in middle-aged men. J Am Coll Cardiol 38:72-79

8. Nakata T, Miyamoto K, Doi A et al (1998) Cardiac death prediction and impaired cardiac sympathetic innervation assessed by MIBG in patients with failing and nonfailing hearts. J Nucl Cardiol 5:579-590

9. Santoro A, Mandreoli M (2014) Chronic renal disease and risk of cardiovascular morbidity-mortality. Kidney Blood Press Res 39:142-146

10. Sasano T, Abraham R, Chang KC et al (2008) Abnormal sympathetic innervation of viable myocardium and the substrate of ventricular tachycardia after myocardial infarction. J Am Coll Cardiol 51:2266-2275

11. Chrapko BE, Jaroszyński AJ, Głowniak A et al (2011) lodine-123 metaiodobenzylguanidine myocardial imaging in haemodialysed patients asymptomatic for coronary artery disease: a preliminary report. Nucl Med Commun 32:515-521

12. Kidney Disease (2012) Improving Global Outcomes (KDIGO) Blood Pressure Work Group. KDIGO clinical practice guideline for the management of blood pressure in chronic kidney disease. Kidney Int Suppl 2:337-414

13. Flotats A, Carrió I, Agostini D, EANM Cardiovascular Committee; European Council of Nuclear Cardiology et al (2010) Proposal for standardization of 1231-metaiodobenzylguanidine (MIBG) cardiac sympathetic imaging by the EANM Cardiovascular Committee and the European Council of Nuclear Cardiology. Eur J Nucl Med Mol Imaging 37:1802-1812

14. Germano G, Kavanagh PB, Waechter P et al (2000) A new algorithm for the quantitation of myocardial perfusion SPECT. I: technical principles and reproducibility. J Nucl Med 41:712-719

15. Kurata C, Wakabayashi Y, Shouda S et al (1995) Enhanced cardiac clearance of iodine-123-MIBG in chronic renal failure. J Nucl Med 36:2037-2043

16. Kurata C, Uehara A, Sugi T et al (2000) Cardiac autonomic neuropathy in patients with chronic renal failure on hemodialysis. Nephron 84:312-319

17. Chrapko BE, Bednarek-Skublewska A, Staśkiewicz G, Książek A (2012) Relationship of haemodialysis therapy duration and cardiac adrenergic system function assessed by iodine-123 metaiodobenzylguanidine imaging in haemodialysed nondiabetic patients. Nucl Med Commun 33:155-163

18. Hartmann F, Ziegler S, Nekolla S et al (1999) Regional patterns of myocardial sympathetic denervation in dilated cardiomyopathy: an analysis using carbon-11 hydroxyephedrine and positron emission tomography. Heart 81:262-700

19. Schwitter J, Wacker CM, Wilke N et al (2013) MR-IMPACT II: Magnetic Resonance Imaging for Myocardial Perfusion Assessment in Coronary artery disease Trial: perfusion-cardiac magnetic resonance vs. single-photon emission computed tomography for the detection of coronary artery disease: a comparative multicentre, multivendor trial. Eur Heart J 34:775-781

20. Annual Data Report, US Renal Data System, USRDS (2012) National Institutes of Health, National Institute of Diabetes and Digestive and Kidney Diseases, Bethesda, MD, USA. DOI: https://doi.org/10.1053/j.ajkd.2012.11.031

21. Wan C, Herzog CA, Zareba W, Szymkiewicz SJ (2014) Sudden cardiac arrest in hemodialysis patients with wearable cardioverter defibrillator. Ann Noninvasive Electrocardiol 19:247-257

22. Jacobson AF, Senior R, Cerqueira MD et al (2010) ADMIRE-HF Investigators. Myocardial iodine-123 meta-iodobenzylguanidine imaging and cardiac events in heart failure. Results of the prospective ADMIRE-HF (AdreView Myocardial Imaging for Risk Evaluation in Heart Failure) study. J Am Coll Cardiol 55:2212-2221

\section{Submit your manuscript to a SpringerOpen ${ }^{\circ}$ journal and benefit from:}

- Convenient online submission

- Rigorous peer review

- Open access: articles freely available online

- High visibility within the field

- Retaining the copyright to your article

Submit your next manuscript at springeropen.com 